\title{
Article \\ Crystal Structure of Nitrilase-Like Protein Nit2 from Kluyveromyces lactis
}

\author{
Chaewon Jin ${ }^{1}$, Hyeonseok Jin ${ }^{1}$, Byung-Cheon Jeong ${ }^{2}{ }^{\circledR}$, Dong-Hyung Cho ${ }^{3}$, Hang-Suk Chun ${ }^{4}$, Woo-Keun Kim ${ }^{4}$ \\ and Jeong Ho Chang $1,5, * \mathbb{D}$
}

1 Department of Biology Education, Kyungpook National University, Daehak-ro 80, Buk-gu, Daegu 41566, Korea; jinchaewon178@gmail.com (C.J.); jinhs@postech.ac.kr (H.J.)

2 Department of Pharmacology, University of Texas Southwestern Medical Center, Dallas, TX 75390, USA; Byung-Cheon.Jeong@utsouthwestern.edu

3 School of Life Sciences, Kyungpook National University, Daegu 41566, Korea; dhcho@knu.ac.kr

4 Biosystem Research Group, Korea Institute of Toxicology, Daejeon 34114, Korea; hangsuk.chun@kitox.re.kr (H.-S.C.); wookkim@kitox.re.kr (W.-K.K.)

5 Department of Biomedical Convergence Science and Technology, Kyungpook National University, Daehak-ro 80, Buk-gu, Daegu 41566, Korea

* Correspondence: jhcbio@knu.ac.kr; Tel.: +82-53-950-5913; Fax: +82-53-950-6809

Citation: Jin, C.; Jin, H.; Jeong, B.-C.; Cho, D.-H.; Chun, H.-S.; Kim, W.-K.; Chang, J.H. Crystal Structure of Nitrilase-Like Protein Nit2 from Kluyveromyces lactis. Crystals 2021, 11, 499. https://doi.org/ 10.3390/ cryst11050499

Academic Editors: Abel Moreno, Kyeong Kyu Kim and T. Doohun Kim

Received: 13 March 2021

Accepted: 21 April 2021

Published: 1 May 2021

Publisher's Note: MDPI stays neutral with regard to jurisdictional claims in published maps and institutional affiliations.

Copyright: (c) 2021 by the authors. Licensee MDPI, Basel, Switzerland. This article is an open access article distributed under the terms and conditions of the Creative Commons Attribution (CC BY) license (https:/ / creativecommons.org/licenses/by/ $4.0 /)$.

\begin{abstract}
The nitrilase superfamily, including 13 branches, plays various biological functions in signaling molecule synthesis, vitamin metabolism, small-molecule detoxification, and posttranslational modifications. Most of the mammals and yeasts have Nit1 and Nit2 proteins, which belong to the nitrilase-like (Nit) branch of the nitrilase superfamily. Recent studies have suggested that Nit1 is a metabolite repair enzyme, whereas Nit2 shows $\omega$-amidase activity. In addition, Nit1 and Nit2 are suggested as putative tumor suppressors through different ways in mammals. Yeast Nit2 (yNit2) is a homolog of mouse Nit1 based on similarity in sequence. To understand its specific structural features, we determined the crystal structure of Nit2 from Kluyveromyces lactis (KlNit2) at $2.2 \AA$ resolution and compared it with the structure of yeast-, worm-, and mouse-derived Nit 2 proteins. Based on our structural analysis, we identified five distinguishable structural features from 28 structural homologs. This study might potentially provide insights into the structural relationships of a broad spectrum of nitrilases.
\end{abstract}

Keywords: Nit2; nitrilase superfamily; $\omega$-amidase; Kluyveromyces lactis

\section{Introduction}

The members of the nitrilase superfamily fulfill various biological roles, such as signaling molecule synthesis, vitamin and coenzyme metabolism, small-molecule detoxification, and posttranslational modifications [1]. Most family members share a common carbon-nitrogen $(\mathrm{CN})$ hydrolase domain, found in plants, animals, fungi, as well as many prokaryotes [2,3]. The members contain a catalytic triad in their active sites consisting of highly conserved cysteine, glutamate, and lysine residues [2]. Despite the name of the superfamily, only one branch exhibits nitrilase activity, whereas others are known as acid amides, secondary amidases, ureas, and carbamates.

Based on a sequence analysis and domain fusion patterns, the members of the nitrilase superfamily could be classified into 13 branches [1], among which the nitrilase-like (Nit) protein is included in the 10th branch, originally categorized as Fhit-related tumor suppression proteins [4]. Two Nit subtypes, Nit1 and Nit2, could be found in the Nitbranch in mammals and yeast, which have $\omega$-amidase activities [2,5]. The mammalian Nit1 protein is a homolog of the yeast Nit2 protein [2]. In mammals, Nit2 shows $\omega$-amidase activity toward $\alpha$-ketoglutaramate, whereas Nit1 has a weaker $\omega$-amidase activity $[6,7]$. Nit2 acts to convert glutamine into $\alpha$-ketoglutaramate, which replenishes $\alpha$-ketoglutarate 
to the tricarboxylic acid (TCA) cycle. Some studies have shown that mammalian Nit1 has recently been assumed as an enzyme that functions in repairing metabolic damage. In the study, it was estimated that transaminase inadvertently produces dGSH and that Nit1 serves as a metabolite repair enzyme that converts dGSH to $\alpha$-ketoglutaramate and cysteinylglycine [5]. The expression of the mammalian Nit1 protein (yeast Nit2 homolog) is separated from that of Fhit. However, the NitFhit Rosetta stone protein could be found in invertebrates [8]. This suggests that Nit1 and Fhit are likely to be involved in the same signaling or metabolic pathway, and their activities could be additive.

Several reports suggest that Nit1 and Nit2 are potent tumor suppressors [4,9-13]. Nit1-knockout mice have accelerated cell proliferation with increased expression of cyclin D1, enhanced survival of cells having DNA damage, and increased incidence of $N$-nitrosomethylbenzylamine-induced tumors [12,14]. However, Nit1 overexpression induces both decreased cell viability and increased caspase-3-dependent apoptosis [14]. Thus, the overexpression of Nit2 in HeLa cells leads to a decrease in cell proliferation and growth through induction of G2 arrest rather than by apoptosis. This also indicates that Nit2 can potentially play a role in tumor suppression [11]. Although Nit1 is involved in tumor suppression in multiple ways, it remains unclear whether these roles are related to its catalytic function [5]. Moreover, a mutation on the conserved active-site cysteine to alanine did not alter the ability of Nit1 to promote apoptosis in human cells.

In this study, to understand the specific structural features of Nit2, we determined the crystal structure of Nit2 from Kluyveromyces lactis (KlNit2) at $2.2 \AA$ resolution and compared the three-dimensional structures of nitrilase superfamily members. Based on the structural analysis of Nit2, we managed to distinguish five structural features including the C-terminal region. In addition, we performed the sequence-based phylogenetic analysis of KlNit2 homologous structures. This study might provide insights into the structural and evolutionary relationships of a broad spectrum of nitrilases.

\section{Materials and Methods}

\subsection{Cloning of Nit2 from Kluyveromyces lactis}

The Nit2 gene was amplified from a Kluyveromyces lactis genomic DNA library through a polymerase chain reaction (PCR), as described previously [15]. Briefly, the amplified fragment was digested with the restriction enzymes NheI and XhoI (R016S and R0075, respectively, Enzynomics, Daejeon, Korea), and ligated into pET28a vectors using T4 DNA ligase (M0202S, Roche, Basel, Germany). The plasmid was then transformed into Escherichia coli (E. coli) strain DH5 $\alpha$, and the transformants were confirmed using a colony PCR. All oligonucleotides used in the study were purchased from Cosmogenetech (Seoul, Korea).

\subsection{Purification of the Recombinant KlNit2 Protein}

The plasmids encoding Kluyveromyces lactis Nit2 (KlNit2) proteins were transformed into the BL21 (DE3) Star E. coli strain. Cells were grown and induced with $0.3 \mathrm{mM}$ isopropyl $\beta$-D-1-thiogalactopyranoside (IPTG; 420322, Calbiochem, Sigma-Aldrich, St. Louis, MO, USA) for $16 \mathrm{~h}$ at $20^{\circ} \mathrm{C}$ in LB medium (L4488, MBcell, Seoul, Korea). The harvested cells were disrupted by ultrasonication. The lysate was bound to Ni-NTA agarose (30230, Qiagen, Hilden, Germany) for $90 \mathrm{~min}$ at $4{ }^{\circ} \mathrm{C}$. After washing with buffer A (200 mM NaCl, $50 \mathrm{mM}$ Tris, $\mathrm{pH}$ 8.0) containing $20 \mathrm{mM}$ imidazole (I5513, Sigma-Aldrich, St. Louis, MO, USA), the bound proteins were eluted with $250 \mathrm{mM}$ imidazole in buffer A. Size exclusion chromatography (SEC) was performed using HiPrep 16/60 Sephacryl S-300 HR (17116701, GE Healthcare, Chicago, IL, USA). The buffer used for SEC contained $150 \mathrm{mM} \mathrm{NaCl}, 2 \mathrm{mM}$ dithiothreitol (DTT; 233155, Calbiochem, Sigma-Aldrich, St. Louis, MO, USA), and $20 \mathrm{mM}$ Tris, $\mathrm{pH}$ 7.5. Following SEC, the protein was stored at $-80^{\circ} \mathrm{C}$ pending crystallization trials.

\subsection{Crystallization}

All crystallization trials were performed at $4{ }^{\circ} \mathrm{C}$ using either sitting-drop or hangingdrop vapor diffusion methods. The crystals for KlNit2 with the $\mathrm{N}$-terminal His ${ }_{6}$ tag grew 
within a day in drops containing equal volumes $(1 \mu \mathrm{L})$ of protein and reservoir solutions (26\% w/v polyethylene glycol (PEG 3350), $0.3 \mathrm{M}$ ammonium tartrate dibasic). To improve the crystals, additional screening was performed using an additive (HR2-428, Hampton Research, Aliso Viejo, CA, USA) and a detergent (HR2-406, from Hampton Research, Research, Aliso Viejo, CA, USA) screening kit. Prior to flash cooling all crystals in liquid nitrogen, $30 \%$ glycerol was added to the reservoir solutions as a cryoprotectant.

\subsection{Data Collection and Structure Determination}

Diffraction datasets were collected at $100 \mathrm{~K}$ on beamline 5C of the Pohang Accelerator Laboratory (PAL, Pohang, Korea) using a Quantum 315 CCD detector (San Jose, CA, USA). Data were processed using the HKL-2000 software suite. The crystals of KlNit2 with the $\mathrm{N}$-terminal $\mathrm{His}_{6}$ tag belonged to the space group $\mathrm{C} 2$ and diffracted to a resolution of $2.2 \AA$. The crystal structures were solved by molecular replacement methods using the Phaser-MR in the PHENIX crystallographic software, version 1.9 (PHENIX, Lawrence Berkeley Laboratory, Berkeley, CA, USA) [16]. The Nit2 from Saccharomyces cerevisiae (PDB ID: 4HG3) was used for the initial search model. The model building was performed using the Wincoot program [17]. The structural models were refined using the Phenix.refine program in the package of PHENIX program. All structural figures were visualized by the graphics program PyMOL (Schrödinger Inc., New York, NY, USA). The details of data collection and applied statistics are provided in Table 1.

Table 1. Data collection and refinement statics for KlNit2.

\begin{tabular}{|c|c|}
\hline & $K l \mathrm{Nit} 2$ \\
\hline \multicolumn{2}{|l|}{ Data collection } \\
\hline Space group & C2 \\
\hline \multicolumn{2}{|l|}{ Cell dimensions } \\
\hline$a, b, c(\AA)$ & $79.0,211.1,89.4$ \\
\hline$\alpha, \beta, \gamma\left({ }^{\circ}\right)$ & $90,112.1,90$ \\
\hline Resolution range $(\AA)$ & $50.0-2.2(2.28-2.20)^{1}$ \\
\hline$R_{\text {merge }}(\%)^{2}$ & $8.9(52.1)$ \\
\hline$I / \sigma I$ & $17.0(2.6)$ \\
\hline Total reflections & 392,121 \\
\hline Unique reflections & $68,506(6799)$ \\
\hline Completeness (\%) & $99.9(99.9)$ \\
\hline Redundancy & $5.7(5.6)$ \\
\hline $\mathrm{CC}_{1 / 2}$ & $99.1(86.2)$ \\
\hline \multicolumn{2}{|l|}{ Structure refinement } \\
\hline Resolution range $(\AA)$ & $45.7-2.2$ \\
\hline No. of reflections & 65,198 \\
\hline$R_{\text {work }}{ }^{3} / R_{\text {free }}(\%)^{4}$ & $16.8 / 22.3$ \\
\hline \multicolumn{2}{|l|}{ No. atoms } \\
\hline Protein & 9700 \\
\hline Water & 543 \\
\hline \multicolumn{2}{|l|}{ RMSD } \\
\hline Bond length (§) & 0.009 \\
\hline Bond angle $\left({ }^{\circ}\right)$ & 0.941 \\
\hline Planar group $(\AA)$ & 0.005 \\
\hline Chiral volume $\left(\AA^{3}\right)$ & 0.057 \\
\hline Average $B$-factor $\left(\AA^{2}\right)$ & 39.4 \\
\hline Protein & 39.2 \\
\hline Solvent & 43.3 \\
\hline \multicolumn{2}{|l|}{ Ramachandran plot (\%) } \\
\hline Favored region & 97.2 \\
\hline Allowed & 2.4 \\
\hline Disallowed & 0.4 \\
\hline PDB code & 7ELF \\
\hline
\end{tabular}

\footnotetext{
${ }_{1}^{1}$ The numbers in parentheses are statistics from the highest-resolution shell. ${ }^{2} R_{\text {merge }}=\Sigma\left|I_{\mathrm{obs}}-I_{\mathrm{avg}}\right| / I_{\mathrm{obs}}$, where $I_{\mathrm{obs}}$ is the observed intensity of individual reflection, and $I_{\mathrm{avg}}$ is averaged over symmetry equivalents. ${ }^{3} R_{\text {work }}=\Sigma|| F_{\mathrm{O}}|-| F_{\mathrm{C}}|| / \Sigma\left|F_{\mathrm{O}}\right|$, where $\left|F_{\mathrm{o}}\right|$ and $\left|F_{\mathrm{C}}\right|$ are the observed and calculated structure factor amplitudes, respectively. ${ }^{4} R_{\text {free }}$ was calculated using $5 \%$ of the data.
} 


\section{Results}

\subsection{Overall Structure of KlNit2}

The KlNit2 protein showed high homology with its homologous proteins such as Saccharomyces cerevisiae Nit2 (ScNit2; sequence identity, 60.32\%), Caenorhabditis elegans Nit-Fhit (ceNitFhit; sequence identity, 33.44\%), and Mus musculus Nit2 (MmNit2; sequence identity, 29.84\%) (Figure 1A).
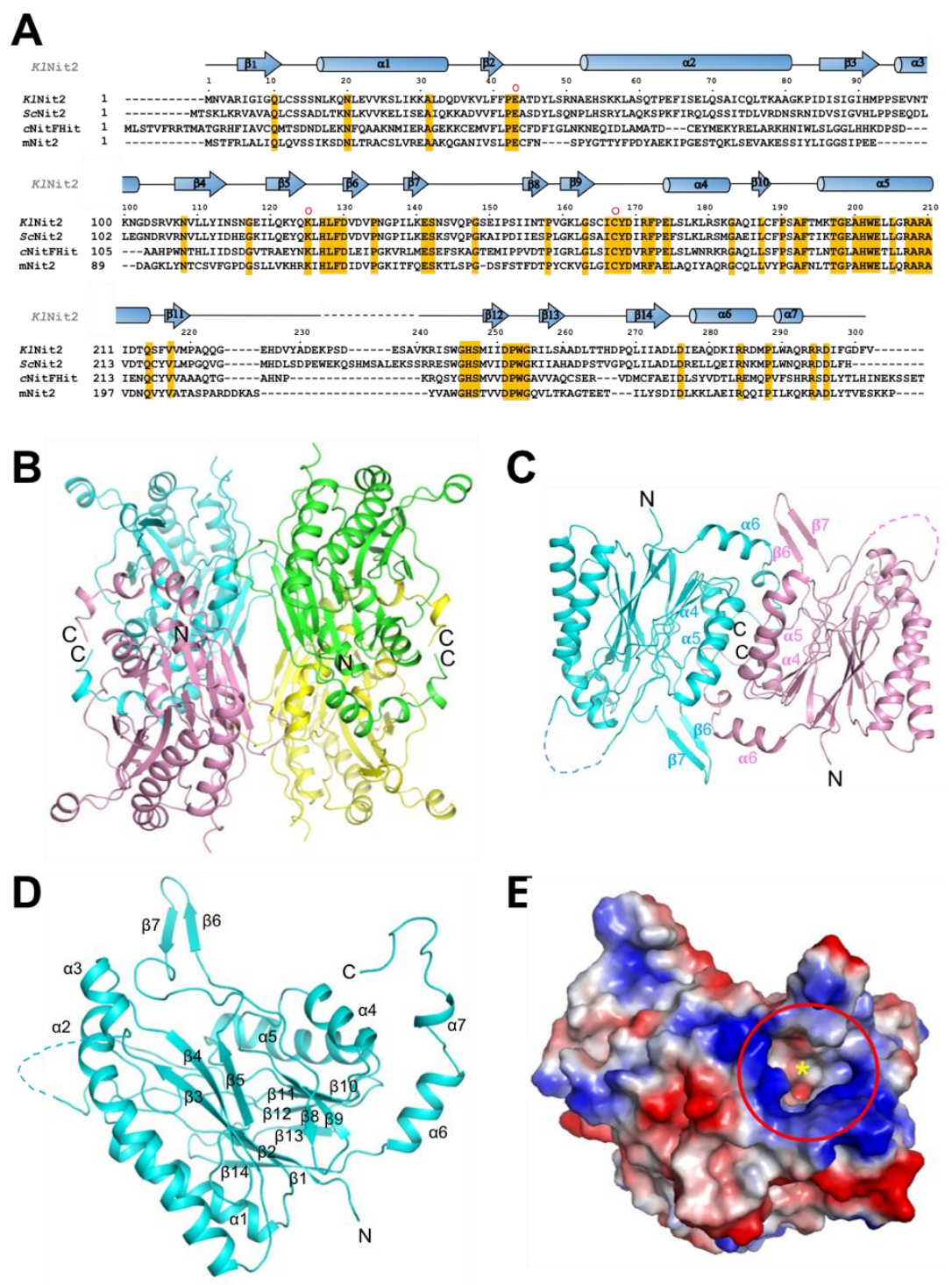

Figure 1. Overall structure of KINit2. (A) Sequence alignment of KINit2, ScNit2, mNit2, and the Nit region of cNitFhit. The secondary structure elements of KINit2 are highlighted at the top of the sequences. Black dots above the sequences indicate every tenth residue in K1Nit2. Identical residues within KINit2 and its homologs are highlighted in yellow and the key residues of the active site are marked by a red circle on the sequences. (B) The tetrameric structure of KINit2 is shown in a ribbon diagram. (C) The dimer structure of KINit2. One monomer is shown in cyan; the other is colored light pink. The dashed lines show the regions that were not visible in the electron density map. (D) The monomer structure of KINit2. The dashed line shows the region that was not visible in the electron density map. (E) The electrostatic surface model of the KINit2 monomer. Red and blue represent the negatively and positively charged surfaces, respectively. The active site is highlighted in a red circle. The figures were generated using the graphics program PyMOL (Schrödinger Inc., New York, NY, USA). 
While the KlNit2 protein forms a dimer in solution [15], four molecules exist in the asymmetric unit (Figure 1B). Dimerizations between subunits I and III as well as subunits II and IV are mediated by their $\alpha 4$ and $\alpha 5$ helices. Although both dimers can be stabilized by $\beta 9-\alpha 4$ and $\beta 12-\beta 13$ loops, such interactions do not seem to be strong for each dimer. More stable dimers are generated by subunit association between neighboring symmetry-related molecules (Figure 1C). The dimeric interface is composed of $\alpha 4, \alpha 5, \alpha 6, \beta 6$, and $\beta 7$ that contribute to extensive interactions, as shown in a previous study of Saccharomyces cerevisiae Nit2 (ScNit2) [18]. The overall structure of KlNit2 has been described as an $\alpha-\beta-\beta-\alpha$ architecture, similar to the structure of other nitrilase superfamily members [3,18-20]. Each subunit is composed of $7 \alpha$-helices $(\alpha 1-\alpha 7)$ and $14 \beta$-strands $(\beta 1-\beta 14), 2$ central $\beta$-sheets consisting of $12 \beta$-strands (except $\beta 6$ and $\beta 7$ ), surrounded by $5 \alpha$-helices (except for $\alpha 6$ and $\alpha 7$ ) (Figure 1D). In addition, protruded $\beta 6$ and $\beta 7$ strands ( $\beta 6-\beta 7$ hairpin) are positioned to cover the vicinity of a deep pocket containing a Lys-Cys-Glu triad, which plays an important role in the amidase activity of the protein (Figure 1D,E).

\subsection{Active Site}

The active site of $K I N i t 2$ is located in the deep pocket with a structure that closely resembles that of the other nitrilase superfamily members, such as mammalian Nit2 [3], yeast Nit2 [18], worm NitFhit Nit domain [8], bacterial Nit2 [20], and archaeal Nit2 [19] (Figure 2A). We observed that the $\beta 6$ and $\beta 7$ strands are localized in the vicinity of the active site. Thus, in the dimeric structure, the $\alpha 6$ and $\alpha 7$ helices from the neighboring subunit could be found near the active site. The distance between the catalytic sulfur of Cys167 and the oxygen atom of Glu43 is within the range of a hydrogen bond (Figure 2B). However, the distance between the sulfur atom of Cys167 and the $\mathrm{N}^{\varepsilon}$ atom of Lys125 is $6.09 \AA$ that is slightly bigger than that observed in the yeast Nit2 structure [18]. It might be due to the ligand $\alpha$-ketoglutarate binding the yeast Nit2 (Figure 3A). Thus, a thioester bond could be formed between the $\gamma$-carboxyl of the substrate and the $-\mathrm{SH}$ part of the active-site cysteine in the yeast Nit2. The distance between the oxygen of Glu43 and the $\mathrm{N}^{\varepsilon}$ atom of Lys125 is $4.63 \AA$, which is similar to that observed in the yeast Nit2 structure. The catalytic residues are well-conserved in the active site (Figure 1A). It is known that the catalytic triad with Ala192 and Tyr167 helps to bind a ligand intermediate [18] (Figure 2C). Thus, the residues Arg171, Thr194, and Thr197 immobilize the $\alpha$-carboxyl group ligand. The aromatic residues Phe129 and Phe193 maintain a hydrophobic environment.
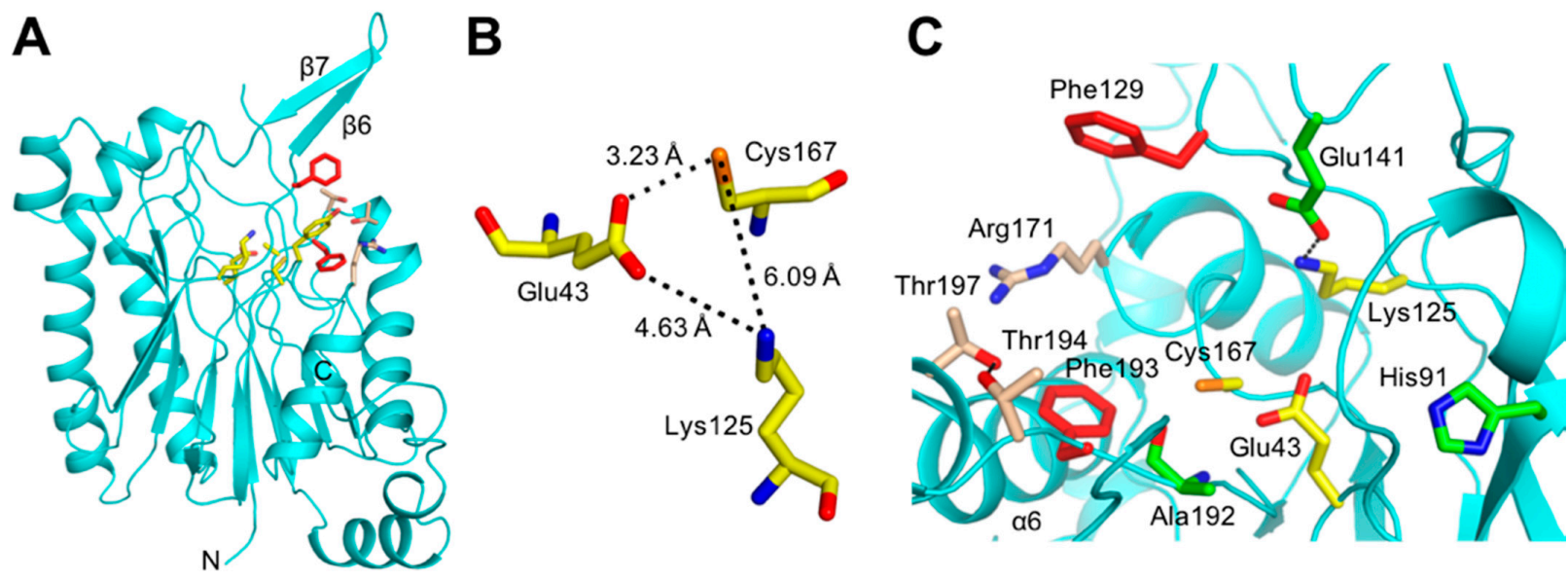

Figure 2. KINit2 active site. (A) Monomeric structure including the active site. (B) The catalytic triad of the active site with distances shown between the key catalytic atoms. (C) The key residues of the active site. The catalytic triad (Glu43-Lys125Cys167) is shown in yellow. The residues (Arg171, Thr194, and Thr197) that immobilize the $\alpha$-carboxyl are shown in the color wheat. The residues (Phe129 and Phe193) that maintain a hydrophobic environment are shown in red. Figures were generated by the graphics program PyMOL (Schrödinger Inc., New York, NY, USA). 

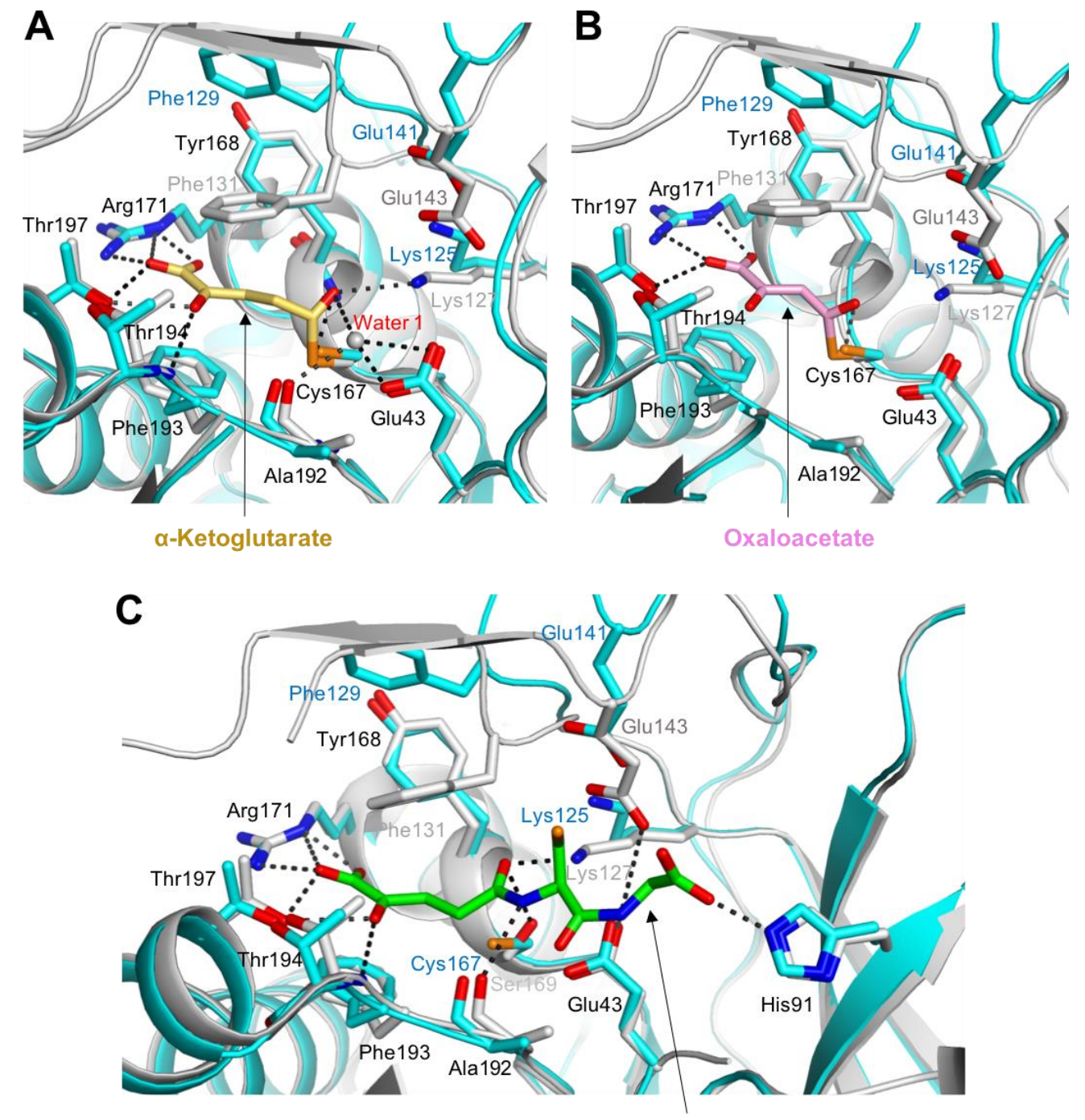

$\mathrm{N}$-(4-carboxy-4-oxobutanoyl)-L-cysteinylglycine

Figure 3. Superposition of the K1Nit2 and ScNit2 ligand complex structures. (A) Detailed view of the interaction between $\alpha$-ketoglutarate and overlaid KINit2 with Nit2 from Saccharomyces cerevisiae (ScNit2; PDB code, 4HG3). Residues from KINit2 with ScNit2 are shown in cyan and gray, respectively; $\alpha$-ketoglutarate is shown in yellow. Water molecule 1 is shown as a gray sphere. (B) Detailed view of the interaction between oxaloacetate (OAA) and overlaid KINit2 with ScNit2 (PDB code, 4HG5). The residues from KINit2 with ScNit2 are shown as in Figure 3A. The OAA is shown in pink. (C) Detailed view of the interaction between a peptide-like ligand and overlaid KINit2 with ScNit2 (PDB code, 4HGD). The residues from KINit2 with ScNit2 are shown as in Figure 3A. The ligand is shown in green. The figures were generated using the graphics program PyMOL (Schrödinger Inc., New York, NY, USA).

\subsection{Ligand-Binding Modes}

To understand the ligand-binding mechanism of KlNit2, we compared the structures of $S c N i t 2$ in complexes with two products: $\alpha$-ketoglutarate ( $\alpha$-KG) (PDB code: $4 \mathrm{HG} 3$ ) and oxaloacetate (OAA) (PDB code: 4HG5) [18]. A structure of the ScNit2 C169S mutant (PDB code: 4HGD) was also reported in complex with an unknown endogenous glutathionelike ligand, $\mathrm{N}$-(4-carboxy-4-oxo-butanoyl)-1-cysteinylglycine (KGT) in the active site. We superimposed these previously published structures on that of KlNit2. While most residues in the active site are well aligned with those of the corresponding residues in ScNit2, several residues showed different conformations. The positions of Phe129 and Glu141 in KlNit2, corresponding to the Phe131 and Glu143 of $S c$ Nit2, significantly changed toward 
the ligands (Figure 3A-C). The Phe131 with $S c$ Nit2 Phe195 covers the thioester region of the ligands in sandwich positions. However, Glu143 does not interact with the two products $\alpha-K G$ and OAA, whereas it interacts with the amide group of KGT (Figure 3C), which might be due to the large movement of the $\beta 6-\beta 7$ hairpin driven by Phe129 (Phe131 of $S c$ Nit2) upon ligand binding (see Section 3.4). In the three ligand-binding structures of ScNit2, Arg173, Thr196, and Thr199 mainly contribute to stabilize the $\alpha$-keto acidic group of the ligands by hydrogen bonds (Figure 3A-C). However, a water molecule mediates interactions between the active-site residues (Glu45, Lys127, and Ala194) and the carbonyl group of $\alpha-\mathrm{KG}$ (Figure 3A). Interestingly, no notable interaction could be found in the OAA complex structure (Figure 3B). In the KGT complex structure, His93 and Glu143 established additional interactions with the amide and carboxylic acid groups (Figure $3 \mathrm{C}$ ). Regarding the active site environment, the ligand-binding modes of KlNit2 might be similar to those of ScNit2.

\subsection{Structural Comparison between KlNit2 and Its Homologs}

To identify structurally varied regions of $K l \mathrm{Nit} 2$, we selected three available homologous structures and superimposed: $S_{c} \mathrm{Nit} 2$ (PDB code, 4H5U), MmNit2 (PDB code, 2W1V), and $\mathrm{CeNitFhit} \mathrm{(PDB} \mathrm{code,} \mathrm{1EMS)}[3,8,18]$. Based on the DALi server output, ScNit2 structure exhibited the most similarity to that of KlNit2 with a Z-score of 45.9 and a root mean square deviation (r.s.m.d.) value of $1.3 \AA$ [21] (Table 2).

Table 2. Structural similarity comparison of homologous structures using Dali ${ }^{1}$.

\begin{tabular}{|c|c|c|c|c|c|c|}
\hline Proteins & Species & Z-Score & $\underset{\text { (A) }}{\text { RMSD }}$ & $\begin{array}{c}\text { Identity } \\
(\%)\end{array}$ & $\mathrm{C}_{\alpha}$ & PDB Code \\
\hline Nit2 & S. cerevisiae & 46.1 & 1.3 & 63 & 292 & 4HG3 \\
\hline NitFhit & C. elegans & 39.1 & 1.5 & 38 & 412 & 1EMS \\
\hline Nit2 & Mus musculus & 33.9 & 2.2 & 32 & 274 & 2W1V \\
\hline Nit3 & S. cerevisiae & 32.3 & 2.1 & 30 & 271 & $1 F 89$ \\
\hline Putative carbon-nitrogen family hydrolase & Staphylococcus aureus & 32.1 & 2.1 & 22 & 268 & $3 \mathrm{P} 8 \mathrm{~K}$ \\
\hline Hypothetical protein Ph0642 & Pyrococcus horikoshii & 32.0 & 2.1 & 23 & 262 & $1 \mathrm{~J} 31$ \\
\hline Hyperthermophilic nitrilase & Pyrococcus abyssi & 31.5 & 2 & 22 & 261 & 3IVZ \\
\hline NitN Amidase & Nesterenkonia sp. AN1 & 31.5 & 2 & 21 & 155 & 5JQN \\
\hline Amidase & Nesterenkonia sp. 10004 & 31.4 & 1.9 & 21 & 255 & $5 N Y B$ \\
\hline Carbon-nitrogen hydrolase & Helicobacter pylori & 31.1 & 1.9 & 24 & 293 & 6MG6 \\
\hline$N$-carbamoyl-D-amino acid amidohydrolase & Agrobacterium sp. & 31 & 2.5 & 30 & 303 & 1ERZ \\
\hline Amidase & Bacillus sp. & 31 & 3 & 17 & 339 & $4 \mathrm{KZF}$ \\
\hline$N$-carbamoyl-D-amino-acid amidohydrolase & Rhizobium radiobacter & 30.9 & 2.5 & 21 & 302 & 2GGK \\
\hline Medicago truncatula $N$-carbamoylputrescine & Medicago truncatula & 30.9 & 2.9 & 22 & 292 & $5 \mathrm{H} 8 \mathrm{I}$ \\
\hline Aliphatic amidase & Pseudomonas aeruginosa & 30.7 & 2.4 & 18 & 341 & 2UXY \\
\hline Bacillus cereus formamidase (BceAmiF) & Bacillus cereus & 30.7 & 2.5 & 17 & 277 & $5 \mathrm{H} 3 \mathrm{O}$ \\
\hline Putative Nit protein & Xanthomonas campestris pv. & 29.9 & 2.5 & 20 & 265 & $2 \mathrm{E} 11$ \\
\hline Glutamine-dependent NAD ${ }^{+}$synthetase & Mycobacterium tuberculosis & 29.9 & 2.2 & 19 & 650 & 3SZG \\
\hline Pyrimidine-degrading enzyme & Drosophila melanogaster & 29.4 & 2.4 & 21 & 379 & $2 \mathrm{VHI}$ \\
\hline Formamidase AmiF & Helicobacter pylori & 29.1 & 2.2 & 19 & 317 & $2 \mathrm{E} 2 \mathrm{~L}$ \\
\hline Glutamine-dependent NAD ${ }^{+}$synthetase & Burkholderia thailandensis & 28.7 & 2.3 & 18 & 540 & $4 \mathrm{~F} 4 \mathrm{H}$ \\
\hline$\beta$-Ureidopropionase & Homo sapiens & 28.4 & 2.6 & 19 & 332 & 6FTQ \\
\hline Nit6803 & Synechocystis sp. & 28.3 & 2.5 & 20 & 287 & 3WUY \\
\hline Glutamine-dependent NAD ${ }^{+}$synthetase & Acinetobacter baumannii & 28.2 & 2.4 & 19 & 526 & 5KHA \\
\hline Nit4 & Arabidopsis thaliana & 28.1 & 2.1 & 23 & 289 & $6 \mathrm{I} 00$ \\
\hline Nh3-dependent NAD ${ }^{+}$synthetase & Streptomyces avermitilis & 27.6 & 2.3 & 19 & 568 & 3N05 \\
\hline Glutamine-dependent NAD ${ }^{+}$synthetase & Cytophaga hutchinsonii & 27.4 & 2.1 & 20 & 580 & 3ILV \\
\hline Vanin-1 & Homo sapiens & 26.6 & 3.5 & 16 & 462 & $4 \mathrm{CYF}$ \\
\hline
\end{tabular}

1 This server computes optimal and suboptimal structural alignments between two protein structures using the DaliLite-pairwise option. http:/ / ekhidna.biocenter.helsinki.fi/dali/ (accessed on 21 April 2021).

Next, the structures of $\mathrm{CeNitFhit}$ and $M m \mathrm{Nit} 2$ exhibited similarity with a Z-score of 39.1 and an r.s.m.d. value of $1.5 \AA$ and $M m$ Nit1with a Z-score of 33.9 and an r.s.m.d. value of $2.2 \AA$, respectively. Overall, we identified several regions, while the four structures shared two central $\beta$-sheets surrounded by five $\alpha$-helices (Figure 4 ). 


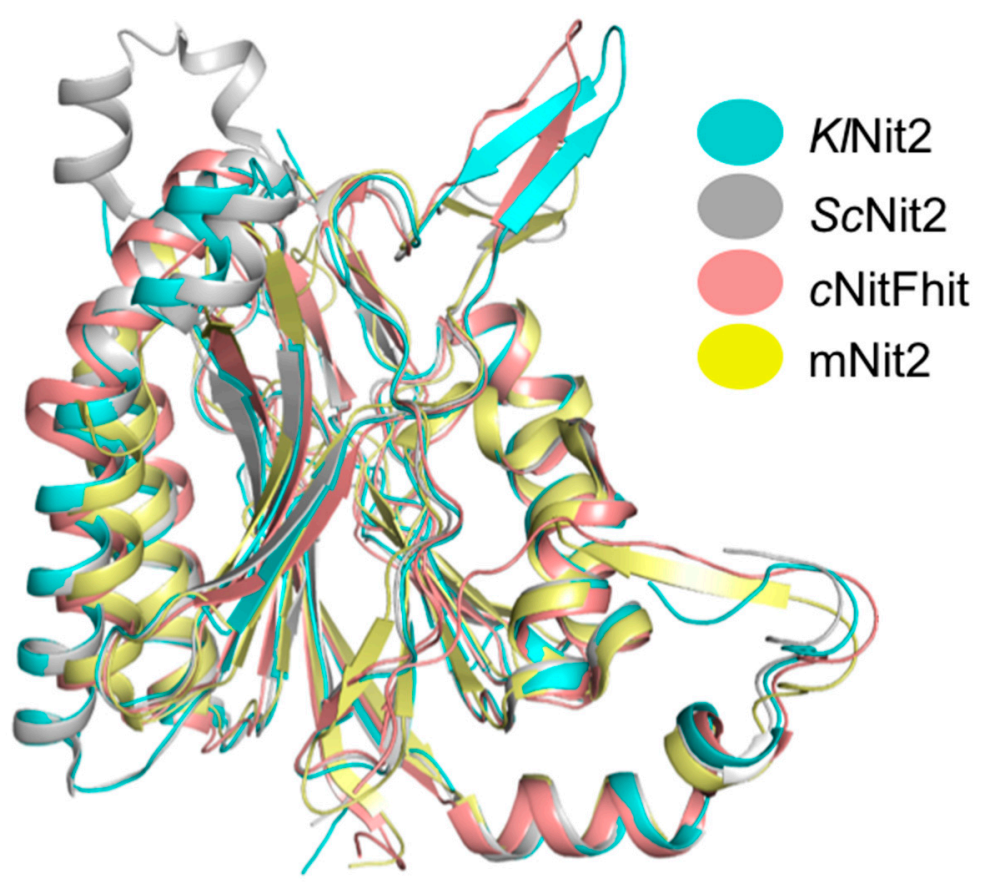

Figure 4. Comparison between KlNit2 and its homologs. Structural superimposition of KlNit2 (cyan) with $S c$ Nit2 (gray), mNit2 (yellow) and with the Nit region of $c$ NitFhit (salmon). Figures were generated using the graphics program PyMOL (Schrödinger Inc., New York, NY, USA).

When comparing KlNit2 with ScNit2, the $\beta 6 / 7$ hairpin structure, which covers the substrate tunnel, shows different angles. The superimposition of the monomer structures of each protein showed that $\beta 6 / 7$ hairpin differs by $48.99^{\circ}$ (Figure $5 \mathrm{~A}$, left panel). Therefore, the $\beta 6 /$ hairpin of KlNit2 moved $16.70 \AA$ outward of the ScNit2 $\beta 6 /$ hairpin. Another difference is that there are two more $\beta$ strands of $S c$ Nit2. In the case of $S c$ Nit2, two more $\beta$ strands can be found between $\beta 11$ and $\beta 12$ of KlNit2 (Figure 5A, right panel). Considering the $S c$ Nit2 structure, we found two major distinct regions due to a crystallographic packing effect (Figure 6).

We observed no significant change of the $\beta 6-\beta 7$ hairpin in CeNitFhit (Figure 5B, left panel). However, we identified no $\alpha 3$ helix, but short-turn loop due to sequence variation. In addition, the loop between $\beta 11$ and $\beta 12$ was significantly shorter than that of KlNit2 (Figure 5B, right panel). While the length of the loop was almost 30 residues in the KINit2, only 15 residues could be identified in $\mathrm{CeNitFhit.} \mathrm{Based} \mathrm{on} \mathrm{the} \mathrm{sequence} \mathrm{alignment,} \mathrm{this}$ common region varied the most among the three KINit2 homologs (Figure 1A).

Several locally distinct regions were found in the $M m N i t 2$ structure. First, similar to the $S c$ Nit2 structure, the $\beta 6-\beta 7$ hairpin rotated toward the active site approximately $47^{\circ}$ compared to the KlNit2 structure (Figure 5C, left panel). Second, similar to the structure of $C e$ NitFhit, the length of the loop between $\beta 11$ and $\beta 12$ was shorter than that in KlNit2 (Figure 5C, right panel). Among the four homologs, $M m$ Nit2 exhibited the shortest loop (Figure 1A). Third, an additional $\beta$-strand was found in its C-terminal end (Figure 5C, right panel). Fourth, the long KlNit2 $\alpha 2$ helix was divided into two helices in the $\mathrm{MmNit} 2$ homolog (Figure 5C, left panel). Finally, similar to the CeNitFhit structure, we could observe no $\alpha 3$ helix but a short-turn loop containing four residues (Figure 5C, left panel). 
A
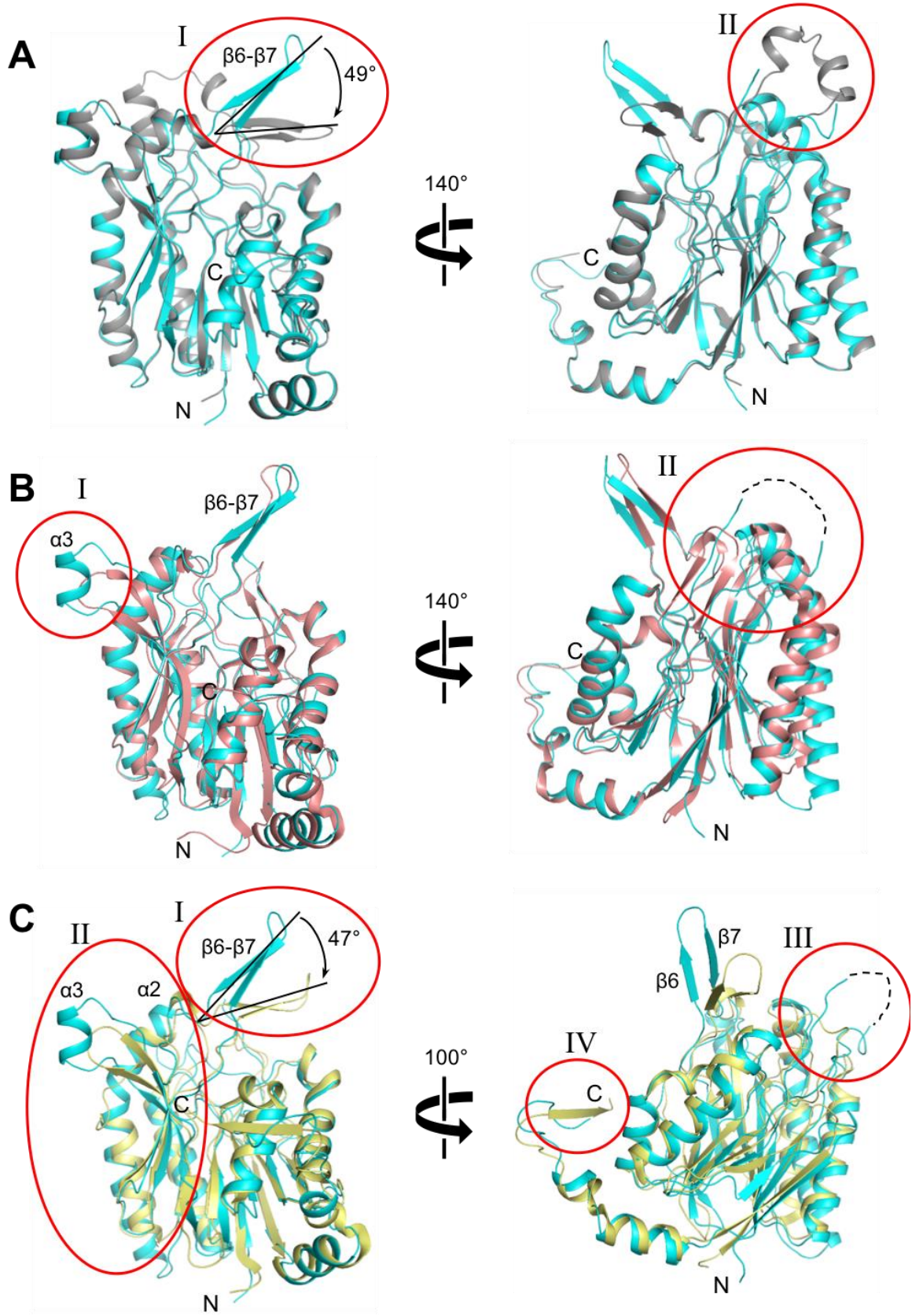

Figure 5. Structural comparison of Nit2 homologs. (A) The overall structures of KINit2 and ScNit2 (PDB code, 4H5U) are depicted in the left panel in cyan and gray, respectively. The right panel shows the view of a $140^{\circ}$ rotation along the $Y$-axis from the orientation compared to the left panel. The two different structural regions (I, II) are indicated by red circles with annotations. (B) The left panel shows the overall structures of KINit2 and NitFhit from Caenorhabditis elegans (cNitFhit; PDB code, 1EMS) in cyan and salmon, respectively. The right panel highlights the view of a $140^{\circ}$ rotation along the $Y$-axis compared to the orientation shown in the left panel. The two different structural regions (I, II) are indicated by red circles with annotations. (C) The overall structures of KINit2 and mouse Nit2 (MmNit2, PDB code, 2W1V) are depicted in the left panel in cyan and yellow, respectively. The right panel shows the view of a $100^{\circ}$ rotation along the $Y$-axis compared to the orientation shown in the left panel. The four different structural regions (I-IV) are indicated by red circles with annotations. The figures were generated using the graphics program PyMOL (Schrödinger Inc., New York, NY, USA). 

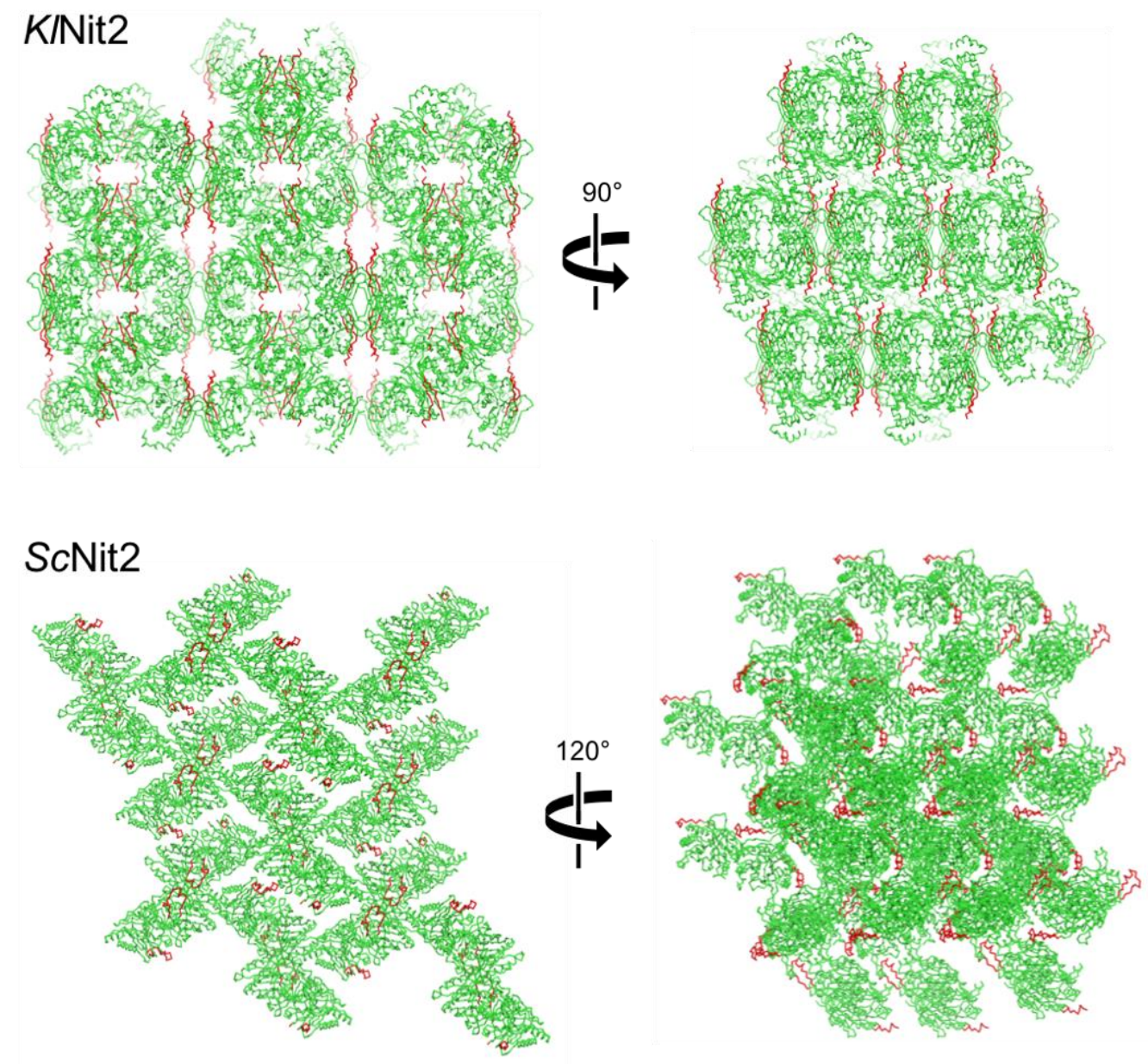

Figure 6. Crystallographic packing of Nit2 proteins. Comparison of crystallographic packing of KINit2 and ScNit2. The figures were generated using the graphics program PyMOL (Schrödinger Inc., New York, NY, USA).

\subsection{Comparison of the Active Site}

The sequence alignment of the KlNit2 homologs showed that most residues exhibiting a relevant activity were highly conserved (Figure 1A). The superimposed structures of KlNit2 with those of $S c \mathrm{Nit} 2$, CeNitFhit, and $\mathrm{MmNit2}$ showed a good overlay in the case of most residues (Figure 7A-C). The overlaid structures of KlNit2 and ScNit2 indicated that most residues interacting with the ligands matched well. However, the positions of Phe131, Glu143, and Lys127 in ScNit2 were located near the catalytic cysteine residue (Figure 7A). As mentioned previously, this effect might be due to conformational difference in the $\beta 6-\beta 7$ hairpin. Likewise, when superimposing the structure of KlNit2 and CeNitFhit, most residues matched well, and even Phe129, Glu141, Lys125 were mostly similar as well (Figure 7B). The overlaid structures of $K l \mathrm{Nit} 2$ and $M m \mathrm{Nit} 2$ showed several different residue conformations (Figure $7 \mathrm{C}$ ). Like the previous sequences, most residues were the same, but His91 in KlNit2 was altered to Pro124 in MmNit2. Although it is not involved in product binding, it might contribute to substrate specificity based on the structure of the ScNit2-KGT complex (Figure 3C). In addition, the conformation of the $\beta 2-\alpha 2$ loop, including Ser49 of KlNit2, changed in MmNit2, containing Tyr87 oriented toward the catalytic cysteine. Phe129 and Glu141 of KlNit2 were also different in MmNit2 similar to that in $\mathrm{ScNit2}$. As a result, these differences might contribute to the smaller active site of $M m$ Nit2 than that of KlNit2. 

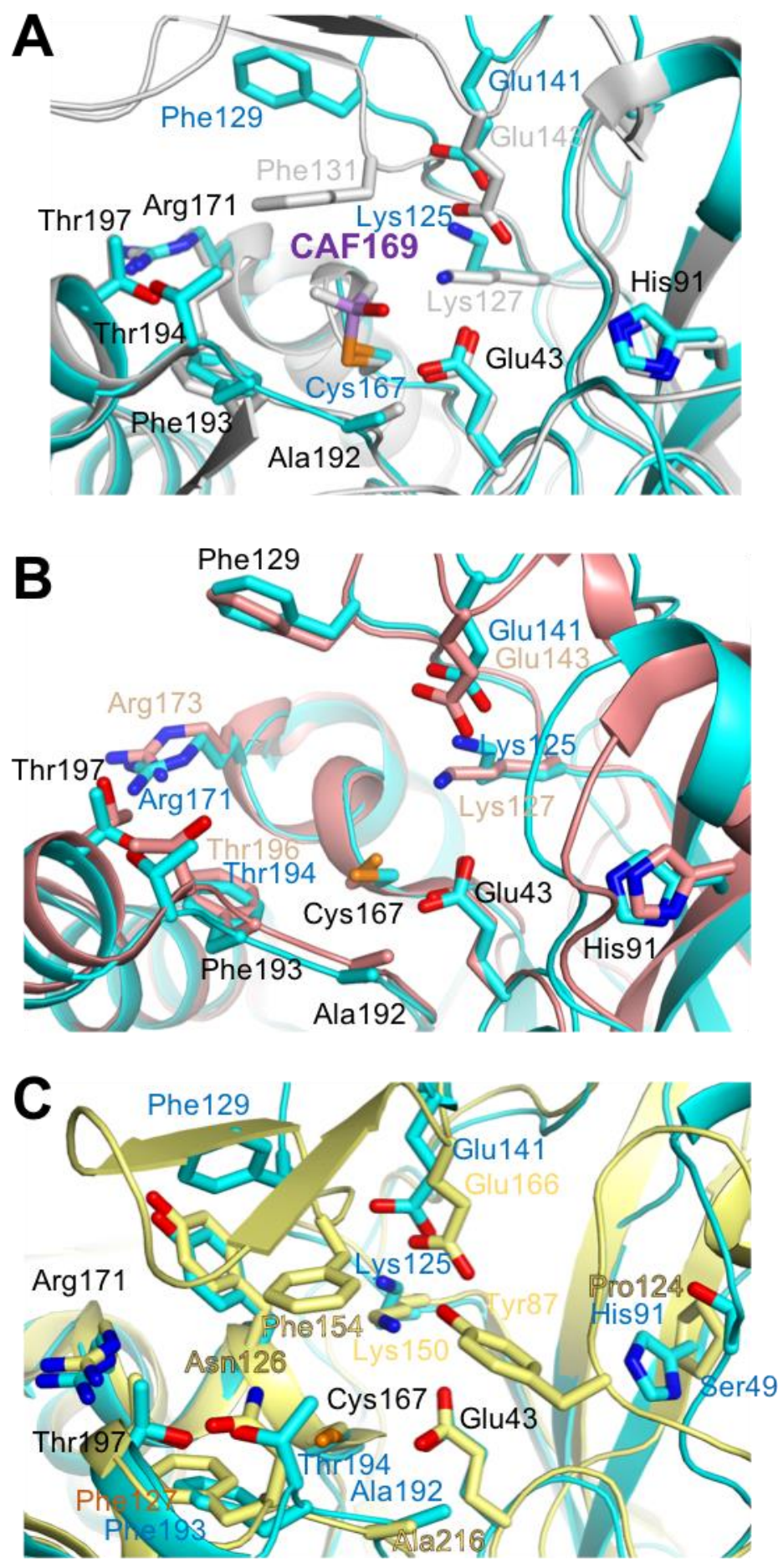

Figure 7. Comparison of the active sites. (A) Overlaid active sites of KINit2 (cyan) and ScNit2 (gray). Residues with less varied conformations are numbered based on KINit2 in black. Residues in distinct conformations are labeled by correspondence structures and colors. The S-dimethylarsinoyl-cystein from ScNit2 is in purple with an annotation. (B) Overlaid active sites of KINit2 (cyan) and cNitFhit (salmon). Residues with less varied conformations are numbered based on KINit2 in black. Residues in distinct conformations are labeled by correspondence structures and colors. (C) Overlaid active sites of KINit2 (cyan) and MmNit2 (yellow). Residues with less varied conformations are numbered based on KINit2 in black. Residues in distinct conformations are labeled by correspondence structures and colors. The figures were generated using the graphics program PyMOL (Schrödinger Inc., New York, NY, USA). 


\subsection{The Structural Features of the KlNit2-Homologous Proteins}

In order to expand our knowledge concerning the structural features in the nitrilase protein family, we searched for KlNit2 structural homologs using the DALI server [21]. Based on the outputs, we could select 28 proteins under a Z-score of 26.6 and an r.s.m.d. value of $3.5 \AA$ (Table 2). The proteins could be divided into ten categories according to their functions. The 22 proteins exhibited 9 functions: nitrilase, $\mathrm{NAD}^{+}$synthetase, nitrilase-like protein (Nit), aliphatic amidase, formamidase, amidohydrolase, beta-ureidopropionase, pyrimidine degrading enzyme, and pantetheinase. Moreover, the 6 proteins also exhibited uncharacterized functions. Following the comparison of the overall structures of 28 proteins, we found 5 specific local conformations. Based on the KlNit2 structure, the five distinct structural features include an $\alpha 2$ helix, a $\beta 3-\alpha 3-\beta 4$ motif, a $\beta 6-\beta 7$ hairpin, $\beta 11-\beta 12$ strands not visible in KlNit2, and a C-terminal part (Figure 8).

The structural feature of the $\alpha 2$ helix was divided into three categories (Figure 8A). While KlNit 2 contains a single $\alpha 2$ helix, partially bent at approximately $132^{\circ}$, the other two types have fragmented helices. The single bent helix feature is widely present among the Nit proteins. The second type showed a deep bent helix of approximately $74^{\circ}$ at the point of two turns from its N-terminal part in NspAN1NitN_Amidase. This feature could also be found in most $\mathrm{NAD}^{+}$synthetases and uncharacterized proteins from Nesterenkonia sp. 10004. The other type exhibited two fully separated helices connected by a loop as shown in PhHp_PH0642.

A

SF I

$(\alpha 2)$
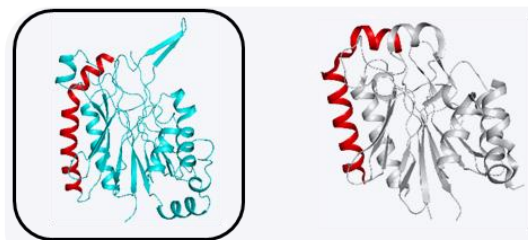

NspAN1NitN_Amidase

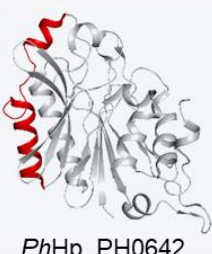

B

$\mathrm{SF}$ II

( $\beta 3-\alpha 4-\beta 4)$
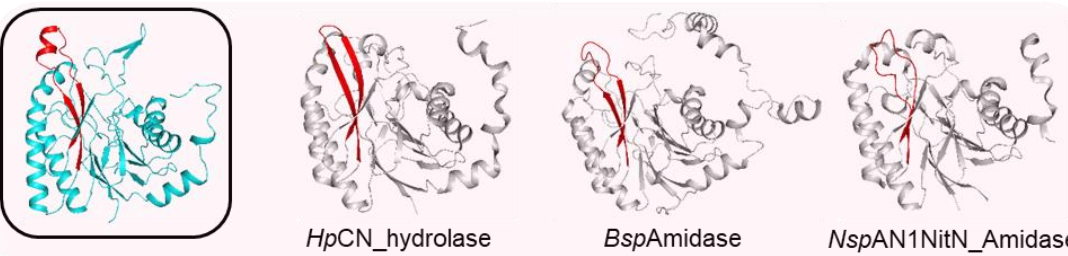

BspAmidase

NspAN1NitN_Amidase

C

SF III

( $\beta 6-\beta 7$

hairpin)

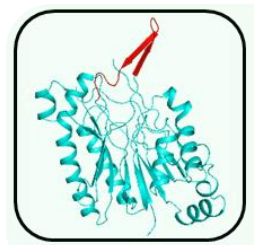

D

SF IV

( $\beta 11-\beta 12)$
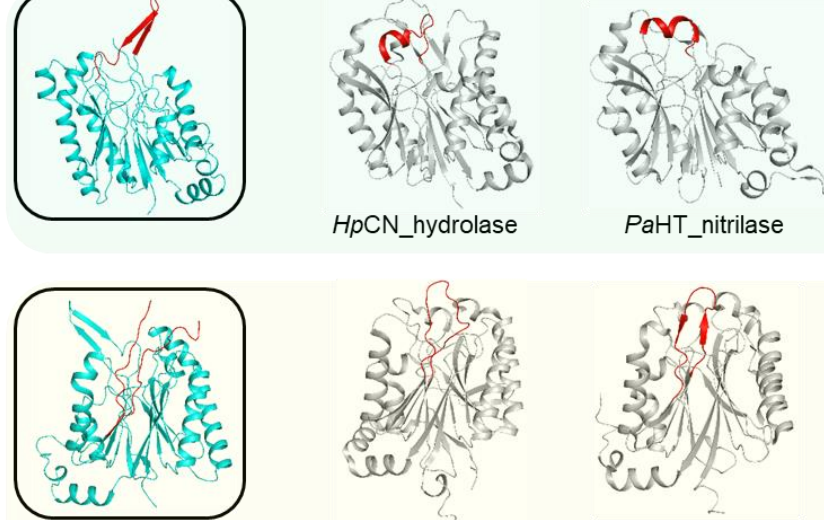

HpCN_hydrolase

PaHT_nitrilase

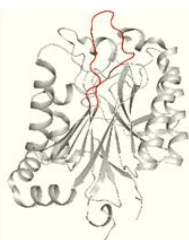

MtGlu-dep_

$\mathrm{NAD}^{+}$synthetase
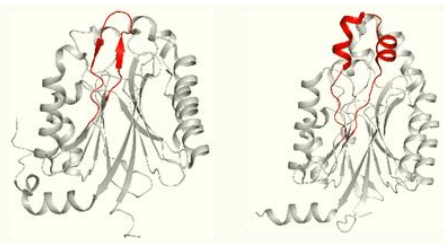

SaPCNF_Hydrolase SspNit6803

Figure 8. Cont. 
E

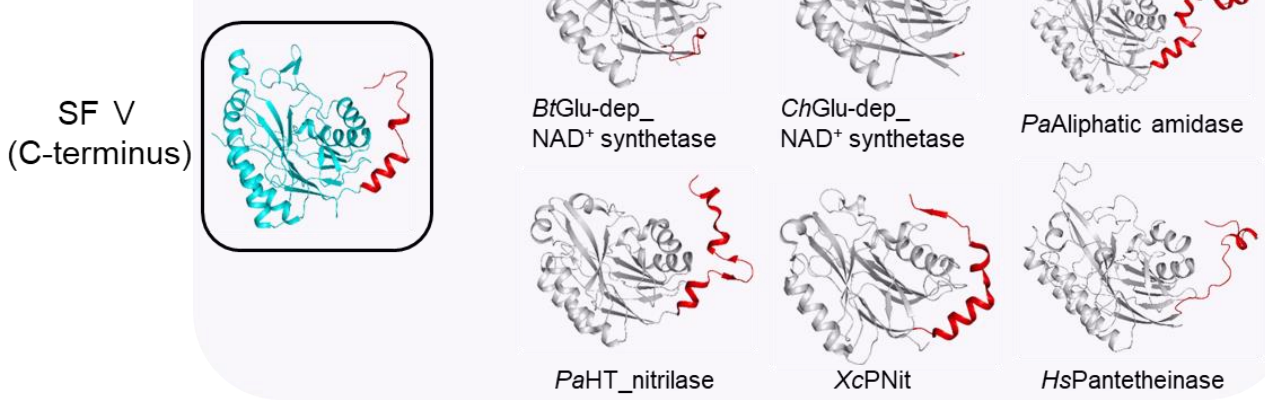

Figure 8. Five distinct structural features. (A) The representative structures containing structural feature I (SF I) according to the $\alpha 2$ helix. The K1Nit2 structure is colored in cyan within a black rectangular box. The two structural homologs with a different conformation of the $\alpha 2$ helix are shown in gray. The $\alpha 2$ helix is shown in red. The two homologous structures indicate the following: NspAN1NitN_Amidase: NitN amidase from Nesterenkonia sp. AN1 (PDB code, 5JQN); PhHp_PH0642: Hypothetical Protein PH0642 from Pyrococcus horikoshii (PDB code, 1J31). (B) The representative structures containing structural feature II (SF II) according to the $\beta 3-\alpha 4-\beta 4$ motif based on KINit2. The KINit2 structure is colored in cyan within a black rectangular box. The three structural homologs with conformations different from $\beta 3-\alpha 4-\beta 4$ are shown in gray. The $\beta 3-\alpha 4-\beta 4$ are shown in red. The three homologous structures indicate the following: HpCN hydrolase: carbon-nitrogen (CN) hydrolase from Helicobacter pylori (PDB code, 6MG6), BspAmidase: Amidase from Bacillus sp. (PDB code, 4KZF), NspAN1NitN_Amidase: NitN amidase from Nesterenkonia sp. AN1 (PDB code, 5JQN). (C) The representative structures containing structural feature III (SF III) according to the $\beta 6-\beta 7$ hairpin. The KINit2 structure is colored in cyan within a black rectangular box. The two structural homologs with different conformations regarding the $\beta 6-\beta 7$ hairpin are shown in gray. The corresponding $\beta 6-\beta 7$ hairpin region is shown in red. The two homologous structures indicate the following: HpCN hydrolase: CN hydrolase from Helicobacter pylori (PDB code, 6MG6), PaHT_nitrilase: hyperthermophilic nitrilase from Pyrococcus abyssi (PDB code, 3KLC). (D) The representative structures containing structural feature IV (SF IV) according to the $\beta 11-\beta 12$ structure. The KINit2 structure is colored in cyan within a black rectangular box. The three structural homologs with a different conformation regarding the $\beta 11-\beta 13$ are shown in gray. The corresponding $\beta 6-\beta 7$ hairpin region is shown in red. The three homologous structures indicate the following: MtGlu-dep_NAD ${ }^{+}$synthetase: glutamine dependent $\mathrm{NAD}^{+}$synthetase from Mycobacterium tuberculosis (PDB code, 3SZG), SaPCNF_Hydrolase: putative CN family hydrolase from Staphylococcus aureus (PDB code, 3P8K), SspNit6803: Nit6803 from Synechocystis sp. (PDB code, 3WUY). (E) The representative structures containing structural feature V (SF V) according to the C-terminus. The KINit2 structure is colored in cyan within a black rectangular box. The six structural homologs with a different conformation regarding the C-terminus are shown in gray. The corresponding C-terminal region is shown in red. The six homologous structures indicate the following: BtGlu-dep_NAD ${ }^{+}$synthetase: glutamine dependent $\mathrm{NAD}^{+}$synthetase from Burkholderia thailandensis (PDB code, 4F4H). ChGlu-dep_NAD ${ }^{+}$synthetase: glutamine dependent $\mathrm{NAD}^{+}$synthetase from Cytophaga hutchinsonii (PDB code, 3ILV), PaAliphatic amidase: aliphatic amidase from Pseudomonas aeruginosa (PDB code, 2UXY), PaHT_nitrilase: hyperthermophilic nitrilase from Pyrococcus abyssi (PDB code, 3KLC), XcPNit: putative Nit from Xanthomonas campestris (PDB code, 2E11), HsPantetheinase: Vanin-1 from Homo sapiens (PDB code, 4CYF). The figures were generated using the graphics program PyMOL (Schrödinger Inc., New York, NY, USA).

Structural feature II contains four types of structures based on the $\alpha 3$ helix between the $\beta 3$ and $\beta 4$ strands shown in KlNit2 (Figure 8B). Except for KlNit2 and ScNit2, the other types have no $\alpha$-helix between the $\beta 3$ and $\beta 4$ strands, and the length of the two strands varied. Amidohydrolase, pyrimidine degrading enzyme, two NAD ${ }^{+}$synthetases, and several Nit proteins exhibit longer strands than $\mathrm{KlNit} 2$. Formamidase, beta-ureidopropionase, nitrilase, pantetheinase, and three $\mathrm{NAD}^{+}$synthetases contain strands of similar length as KlNit2. Shorter strands could be found in two Nesterenkonia amidases.

The third feature, the $\beta 6-\beta 7$ hairpin structure, could be divided into three types (Figure $8 \mathrm{C}$ ). Most Nit proteins, including KlNit2, have a clear $\beta 6-\beta 7$ hairpin. Other types contain no $\beta$ strands and have one or two turns of $\alpha$-helix with a loop instead. A protruding loop with a single turn of $\alpha$-helix could be found in amidohydrolase, pyrimidine 
degrading enzymes, and $\mathrm{NAD}^{+}$synthetase. Proteins with a two-turn $\alpha$-helix include two amidohydrolases, formamidase, nitrilase, pantetheinase, and aliphatic amidase.

We were not able to determine the local conformation between the $\beta 11$ and $\beta 12$ strands in $\mathrm{KlNit} 2$ due to the lack of electron density. Regarding this feature, we made three categories, except for a protein that failed to reveal the similar region as observed in KlNit2 (Figure 8D). The first type showed entirely the loop found in ScNit3, MmNit2, Mycobacterium tuberculosis glutamine-depending $\mathrm{NAD}^{+}$synthetase, and pantetheinase. The second type exhibited a hairpin structure with two $\beta$ strands found in most proteins, such as amidohydrolase, formamidase, aliphatic amidase, and $\mathrm{NAD}^{+}$synthetase. The last type involved two $\alpha$-helices connected by loops found in Synechocystis sp. Nit6803.

The C-terminal region showed the most variability and could be divided into seven types among the KlNit2 structural homologs (Figure 8E). The first type contained an $\alpha$-helix or winding loop after the $\alpha 6$ helix shown in KlNit2, other Nit proteins, amidohydrolase, pyrimidine degrading enzyme, amidase from Nesterenkonia, and several uncharacterized hydrolases. Second, a $\beta$-strand instead of the $\alpha$-helix after the $\beta 14$ strand was included in three $\mathrm{NAD}^{+}$synthetases. The third type showed no additional structure after the $\beta 14$, similar to the one in ChGlu-dependent $\mathrm{NAD}^{+}$synthetase. The fourth type concerned proteins with a long C-terminal structure including five $\alpha$-helices after the $\beta 14$ that could be found in formamidases and aliphatic amidases. The fifth type contained a $\beta$-hairpin between the two $\alpha$-helices shown in Pyrococcus abyssi aliphatic amidase and uncharacterized nitrilase-related protein PH0642. The sixth type represented a $\beta$-strand after the $\alpha$-helix shown in Nit4, one of $\mathrm{NAD}^{+}$synthetases, and putative Nit proteins. The final type is a structure containing one two-turn $\alpha$-helix with a long loop shown in pantetheinase.

\section{Discussion}

Glutathione (GSH) is composed of a tripeptide ( $\gamma$-glutamylcysteinylglycine), which could serve as a potential alternative substrate for various enzymes involved in amino acid and protein metabolism [22]. The $\gamma$-glutamyl moiety of the GSH is a structural analog of glutamate that acts as a common amine donor for aminotransferases [23]. Both the cytosolic and mitochondrial aminotransferases in mammals could produce deaminated glutathione (dGSH) by deaminating the amino group of the GSH $\gamma$-glutamyl moiety [5]. Potentially harmful compounds, such as dGSH, should be eliminated or transformed into other useful compounds. In that sense, mammalian Nit1 plays an important role in converting dGSH by aminotransferase as a useful metabolite. In addition, Arabidopsis Nit1 enzymes extracted from leaves also have an activity for converting GSH into dGSH in the presence of glyoxylate as an amino acceptor [24]. The mammalian Nit1 protein is a homolog of the yeast Nit2 protein [2].

The overall fold of KlNit2 mainly exhibits an $\alpha-\beta-\beta-\alpha$ architecture, similar to those of homologous structures. However, when we expand our comparison to other structures of the Nit family proteins, five distinct structural features, including an $\alpha 2$ helix, a $\beta 3-\alpha 3-\beta 4$, $\beta 6-\beta 7$ hairpin, a $\beta 11-\beta 12$ structure, and a $C$-terminal region, could be found that might potentially contribute to molecular evolution. Considering the $S c$ Nit2 structure, we found two major distinct regions, where KlNit2 exhibited a more extended $\beta 6-\beta 7$ hairpin and disordered $\alpha$-helices between the $\beta 11$ and $\beta 12$ strands due to a crystallographic packing effect (Figure 6). This region might be considered as a framework for a structure-based functional study.

In the KlNit2 active site, most residues, including the catalytic triads, existed in locations similar to that of $S c$ Nit2. However, the active sites of $S c$ Nit2 and MmNit2 were larger than that of $K l \mathrm{Nit} 2$, which might indicate different substrate specificities $[3,18]$. Interestingly, the Teng group unexpectedly found an unidentified ligand as an $\alpha$-keto analog of GSH in the active site of the ScNit2 C169S mutant [18]. They assumed that the molecule could be derived from E. coli. Regarding their finding, we also tested whether the KlNit2 C167A mutant could be complexed with the GSH-like molecule. However, we 
could not obtain any ligand in the active site of the KlNit2 C167S mutant. This might be due to the difference in the size of the substrate binding cavity between KlNit2 and ScNit2.

In summary, we determined the crystal structure of Nit2 from a fungal species Kluyveromyces lactis at a resolution of $2.2 \AA$. Our extensive comparative analysis of KlNit 2 and its structural homologs revealed five distinct structural features and differences in the size of their active sites. This study could potentially provide new insights into the structural relationships among Nit2 family proteins. Further investigation would be required to reveal the sequence-based molecular structural aspect of their evolution.

Author Contributions: Conceptualization, J.H.C.; methodology, B.-C.J.; investigation, C.J., D.-H.C., H.-S.C., W.-K.K., and J.H.C.; data curation, C.J. and H.J.; writing-original draft preparation, C.J. and J.H.C.; writing-review and editing, C.J. and J.H.C.; visualization, C.J. and J.H.C.; supervision, J.H.C.; and funding acquisition, J.H.C. All authors have read and agreed to the published version of the manuscript.

Funding: This research was supported by Basic Science Research Program through the National Research Foundation of Korea funded by the Ministry of Science and ICT (grant No. NRF-2019R1A2C4069796), and from a cooperation project funded by Korea Institute of Toxicology (KK-2101-01) to J.H.C.

Data Availability Statement: Data is contained within the article.

Acknowledgments: We would like to thank the beamline staff Yeon-Gil Kim and Sung Chul Ha at beamlines 5C and 7A of the Pohang Accelerator Laboratory (Pohang, Korea) for data collection.

Conflicts of Interest: The authors declare no conflict of interest.

\section{References}

1. Brenner, C. Catalysis in the nitrilase superfamily. Curr. Opin. Struct. Biol. 2002, 12, 775-782. [CrossRef]

2. Pace, H.C.; Brenner, C. The nitrilase superfamily: Classification, structure and function. Genome Biol. 2001, 2, REVIEWS0001. [CrossRef] [PubMed]

3. Barglow, K.T.; Saikatendu, K.S.; Bracey, M.H.; Huey, R.; Morris, G.M.; Olson, A.J.; Stevens, R.C.; Cravatt, B.F. Functional proteomic and structural insights into molecular recognition in the nitrilase family enzymes. Biochemistry 2008, 47, 13514-13523. [CrossRef] [PubMed]

4. Huebner, K.; Saldivar, J.C.; Sun, J.; Shibata, H.; Druck, T. Hits, Fhits and Nits: Beyond enzymatic function. Adv. Enzym. Regul. 2011, 51, 208-217. [CrossRef] [PubMed]

5. Peracchi, A.; Veiga-Da-Cunha, M.; Kuhara, T.; Ellens, K.W.; Paczia, N.; Stroobant, V.; Seliga, A.K.; Marlaire, S.; Jaisson, S.; Bommer, G.T.; et al. Nit1 is a metabolite repair enzyme that hydrolyzes deaminated glutathione. Proc. Natl. Acad. Sci. USA 2017, 114, E3233-E3242. [CrossRef]

6. Maras, B.; Barra, D.; Duprè, S.; Pitari, G. Is pantetheinase the actual identity of mouse and human vanin-1 proteins? FEBS Lett. 1999, 461, 149-152. [CrossRef]

7. Jaisson, S.; Veiga-da-Cunha, M.; van Schaftingen, E. Molecular identification of omega-amidase, the enzyme that is functionally coupled with glutamine transaminases, as the putative tumor suppressor Nit2. Biochimie 2009, 91, 1066-1071. [CrossRef]

8. Pace, H.; Hodawadekar, S.; Draganescu, A.; Huang, J.; Bieganowski, P.; Pekarsky, Y.; Croce, C.; Brenner, C. Crystal structure of the worm NitFhit Rosetta Stone protein reveals a Nit tetramer binding two Fhit dimers. Curr. Biol. 2000, 10, 907-917. [CrossRef]

9. Mittag, S.; Valenta, T.; Weiske, J.; Bloch, L.; Klingel, S.; Gradl, D.; Wetzel, F.; Chen, Y.; Petersen, I.; Basler, K.; et al. A novel role for the tumour suppressor Nitrilase1 modulating the Wnt/beta-catenin signalling pathway. Cell Discov. 2016, 2, 15039. [CrossRef]

10. Wang, Y.A.; Sun, Y.; le Blanc, J.M.; Solomides, C.; Zhan, T.; Lu, B. Nitrilase 1 modulates lung tumor progression in vitro and in vivo. Oncotarget 2016, 7, 21381-21392. [CrossRef] [PubMed]

11. Lin, C.-H.; Chung, M.-Y.; Chen, W.-B.; Chien, C.-H. Growth inhibitory effect of the human NIT2 gene and its allelic imbalance in cancers. FEBS J. 2007, 274, 2946-2956. [CrossRef]

12. Semba, S.; Han, S.-Y.; Qin, H.R.; McCorkell, K.A.; Iliopoulos, D.; Pekarsky, Y.; Druck, T.; Trapasso, F.; Croce, C.M.; Huebner, K. Biological functions of mammalian Nit1, the counterpart of the invertebrate NitFhit Rosetta stone protein, a possible tumor suppressor. J. Biol. Chem. 2006, 281, 28244-28253. [CrossRef]

13. Zhang, H.; Hou, Y.-J.; Han, S.-Y.; Zhang, E.C.; Huebner, K.; Zhang, J. Mammalian nitrilase 1 homologue Nit1 is a negative regulator in T cells. Int. Immunol. 2009, 21, 691-703. [CrossRef]

14. Lin, C.; Zhang, J.; Lu, Y.; Li, X.; Zhang, W.; Zhang, W.; Lin, W.; Zheng, L.; Li, X. NIT1 suppresses tumour proliferation by activating the TGFbeta1-Smad2/3 signalling pathway in colorectal cancer. Cell Death Dis. 2018, 9, 263. [CrossRef]

15. Jin, C.; Jin, H.; Quade, B.; Chang, J.H. Purification, crystallization, and X-ray crystallographic analysis of Nit2 from Kluyveromyces lactis. Biodesign 2020, 8, 20-23. [CrossRef] 
16. Adams, P.D.; Afonine, P.V.; Bunkóczi, G.; Chen, V.B.; Davis, I.W.; Echols, N.; Headd, J.J.; Hung, L.-W.; Kapral, G.J.; GrosseKunstleve, R.W.; et al. PHENIX: A comprehensive Python-based system for macromolecular structure solution. Acta Crystallogr. D Biol. Crystallogr. 2010, 66 Pt 2, 213-221. [CrossRef]

17. Emsley, P.; Cowtan, K. Coot: Model-building tools for molecular graphics. Acta Crystallogr. D Biol. Crystallogr. 2004, 60, $2126-2132$. [CrossRef] [PubMed]

18. Liu, H.; Gao, Y.; Zhang, M.; Qiu, X.; Cooper, A.J.L.; Niu, L.; Teng, M. Structures of enzyme-intermediate complexes of yeast Nit2: Insights into its catalytic mechanism and different substrate specificity compared with mammalian Nit2. Acta Crystallogr. D Biol. Crystallogr. 2013, 69 Pt 8, 1470-1481. [CrossRef]

19. Sakai, N.; Tajika, Y.; Yao, M.; Watanabe, N.; Tanaka, I. Crystal structure of hypothetical protein PH0642 from Pyrococcus horikoshii at $1.6 \mathrm{~A}$ resolution. Proteins 2004, 57,869-873. [CrossRef]

20. Wang, W.-C.; Hsu, W.-H.; Chien, F.-T.; Chen, C.-Y. Crystal structure and site-directed mutagenesis studies of N-carbamoyl-Damino-acid amidohydrolase from Agrobacterium radiobacter reveals a homotetramer and insight into a catalytic cleft. J. Mol. Biol. 2001, 306, 251-261. [CrossRef] [PubMed]

21. Holm, L. DALI and the persistence of protein shape. Protein Sci. 2020, 29, 128-140. [CrossRef] [PubMed]

22. Forman, H.J.; Zhang, H.; Rinna, A. Glutathione: Overview of its protective roles, measurement, and biosynthesis. Mol. Asp. Med. 2009, 30. [CrossRef] [PubMed]

23. Walker, M.C.; van der Donk, W.A. The many roles of glutamate in metabolism. J. Ind. Microbiol. Biotechnol. 2016, 43, 419-430. [CrossRef] [PubMed]

24. Niehaus, T.D.; Patterson, J.A.; Alexander, D.C.; Folz, J.S.; Pyc, M.; MacTavish, B.S.; Bruner, S.D.; Mullen, R.T.; Fiehn, O.; Hanson, A.D. The metabolite repair enzyme Nit1 is a dual-targeted amidase that disposes of damaged glutathione in Arabidopsis. Biochem. J. 2019, 476, 683-697. [CrossRef] 